\title{
Parâmetros genéticos para características de tamanho e condição corporal, eficiência reprodutiva e longevidade em fêmeas da raça Canchim
}

\author{
Fernando Baldi ${ }^{1}$, Maurício Mello de Alencar ${ }^{2,3}$, Alfredo Ribeiro de Freitas ${ }^{2,3}$, Rogério \\ Taveira Barbosa ${ }^{2}$
}

\footnotetext{
1 UNESP, Campus de Jaboticabal, CEP: 14884-900, Jaboticabal, SP.

2 Embrapa Pecuária Sudeste, CEP: 13560-970, São Carlos, SP.

${ }^{3}$ Bolsista do CNPq.
}

RESUMO - Objetivou-se estimar a herdabilidade das características tempo de permanência no rebanho (TPR) e idades ao primeiro (IDPP), ao segundo (IDSP) e ao terceiro (IDTP) parto e as correlações genéticas entre essas características e os pesos ajustado e não-ajustado para condição corporal, índice de tamanho corporal e condição corporal à primeira monta, ao primeiro parto e à idade adulta. Os componentes de variância e de co-variância foram estimados pelo método de máxima verossimilhança restrita livre de derivadas, utilizando-se análises uni e bicaracterísticas. Os modelos estatísticos incluíram o efeito genético aditivo direto, como aleatório, e o efeito fixo de grupo de contemporâneos. As estimativas de herdabilidade obtidas das análises unicaracterísticas foram 0,10 \pm 0,05 (IDPP); 0,08 \pm 0,05 (IDSP); 0,14 \pm 0,06 (IDTP) e 0,06 \pm 0,00 (TPR), e indicam que provavelmente as características apresentarão baixa resposta à seleção. A seleção para maior peso à idade adulta acarretará redução no tempo de permanência da fêmea no rebanho. A seleção para maiores pesos da fêmea à primeira monta, ao primeiro parto e à idade adulta não deve alterar significativamente as idades ao primeiro, ao segundo e ao terceiro parto. A seleção para os índices de tamanho corporal ou pesos ajustados para condição corporal provavelmente apresentará repostas correlacionadas nas características reprodutivas e de longevidade com tendências semelhantes, mas de maior magnitude em relação à seleção para peso sem ajuste.

Palavras-chave: bovinos de corte, idade ao parto, parâmetros genéticos, pesos, tamanho da vaca, tempo de permanência no rebanho

\section{Genetic parameters for body size, condition score, reproductive and longevity traits in females of the Canchim breed}

\begin{abstract}
The objectives of this study were to estimate heritability for culling age (TPR), ages at first (IDPP), at second (IDSP) and at third calving (IDTP) and genetic correlations of these traits with adjusted and unadjusted weights for condition score, body size indexes and condition score at first mating, at first calving and at mature age. Variance and covariance components were estimated by REML using univariate and bivariate models including the fixed effect of contemporary group and additive direct genetic and residual as random effects. Heritability estimates obtained from univariate analyses were $0.10 \pm 0.05$ (IDPP), $0.08 \pm 0.05$ (IDSP), $0.14 \pm 0.06$ (IDTP) and $0.06 \pm 0.00$ (TPR) and indicate low response to selection on these traits. Genetic correlation estimates suggest that selection for increasing mature weight will reduce culling age but for heavier weight at first mating, at first calving and at maturity will not significantly change ages at first, at second and at third calving. Selection on body size indexes and on cow weights adjusted for condition score may result in similar correlated responses for reproductive traits and longevity which would be higher than those from selection on unadjusted cow weights.
\end{abstract}

Key Words: age at calving, beef cattle, body weight, cow size, days in herd, genetic parameters

\section{Introdução}

As características reprodutivas são as principais determinantes da eficiência biológica e econômica dos sistemas de produção de carne bovina. Na última década, algumas características relacionadas à eficiência reprodutiva foram incorporadas nos programas de avaliação genética de bovinos de corte do Brasil (Alencar, 2002). No entanto, existe maior dificuldade em determinar características facilmente mensuráveis nas fêmeas e geneticamente relacionadas à fertilidade (Johnston \& Bunter, 1996). 
Entre as características reprodutivas de fêmeas, a idade ao primeiro parto em bovinos de corte determina a precocidade reprodutiva do animal. A redução da idade ao primeiro parto aumentará o rendimento econômico proporcionado pelos animais (Rovira, 1996). Outra característica importante de fêmeas é o tempo de permanência da matriz no rebanho, que indica sua habilidade reprodutiva, uma vez que matrizes que apresentam falhas na reprodução tendem a ser descartadas mais cedo (Mercadante et al., 2000).

Apesar da importância das características reprodutivas, o peso corporal ou a taxa de crescimento a idades jovens ainda é o critério de seleção mais utilizado pela maioria dos criadores de bovinos de corte no Brasil. A seleção para maiores pesos e taxas de crescimento em idades jovens pode aumentar o tamanho à idade adulta dos animais (Silva et al., 2000; Talhari et al., 2003; Castro-Pereira et al., 2007). No Brasil, são escassos os trabalhos realizados para estimação da correlação genética entre o peso ou o tamanho da vaca e características reprodutivas e de longevidade; na maioria dos trabalhos (Mercadante et al., 2000; Gianlorenço et al., 2003; Mercadante et al., 2003; Talhari et al., 2003; Bertazzo et al., 2004; Mello et al., 2006; Castro-Pereira et al., 2007), são utilizados pesos a idades jovens, tanto em machos quanto em fêmeas.

Outro aspecto a considerar é a condição corporal da vaca ao parto. Resultados de diversos estudos têm comprovado forte associação entre condição corporal da vaca ao parto e as características reprodutivas (Wiltbank et al., 1962). São escassos na literatura os trabalhos (Vargas et al., 1999; Mercadante et al., 2003) para estudar de forma conjunta as características condição corporal, eficiência reprodutiva e tamanho da matriz.

Portanto, neste trabalho, os objetivos foram estimar as associações genéticas das idades ao primeiro, segundo e terceiro partos e do tempo de permanência da vaca no rebanho com a condição corporal, os pesos ajustado e nãoajustado para condição corporal e o índice de tamanho corporal à primeira monta, ao primeiro parto e à idade adulta.

\section{Material e Métodos}

Os dados utilizados neste trabalho são provenientes do rebanho da raça Canchim da Embrapa Pecuária Sudeste, localizada no município de São Carlos, São Paulo. Os animais desse rebanho foram criados em regime exclusivo de pastagens com suplementação mineral durante todo o ano.

O manejo reprodutivo do rebanho variou durante o período de coleta dos dados utilizados neste trabalho. Até o ano de 1975, as novilhas entravam em reprodução com aproximadamente 34 meses de idade e $360 \mathrm{~kg}$ de peso vivo (PV); a partir de 1976, esses critérios foram mudados para 24 a 28 meses e $300 \mathrm{~kg}$ de PV. Em geral, as vacas eram colocadas com touros após a desmama dos bezerros, ou seja, 7 a 8 meses após a parição, critério modificado em 1969, quando todas as vacas que pariram antes da estação de monta foram colocadas em reprodução. Nova mudança ocorreu a partir de 1976, quando todas as vacas foram colocadas com touros durante a estação de monta, com exceção daquelas prenhes da estação anterior e que iriam parir após o término da estação vigente. Em vários anos, foram utilizadas duas estações de monta, uma no primeiro semestre e outra no segundo. No entanto, estas estações não tinham mês fixo para início ou término e sua duração também variava de 2 a 4 meses. A eliminação de vacas do rebanho ocorreu principalmente por motivos de doença e/ ou acidente; contudo, a partir de 1977, iniciou-se o descarte de vacas consideradas de fertilidade mais baixa, ou seja, que permaneceram não-prenhes por duas estações de monta consecutivas. A partir de 1979, além do peso ao parto, foram avaliadas também quanto à condição corporal ao parto utilizando-se um sistema de pontos: muito magra (escore 3), magra (escore 4), enxuta (escore 5), gorda (escore 6), muito gorda (escore 7) e excessivamente gorda (escore 8).

Neste trabalho foram estudados as idades ao primeiro (IDPP), segundo (IDSP) e terceiro (IDTP) partos e o tempo de permanência no rebanho (TPR, idade em dias em que o animal foi descartado do rebanho). Para TPR, foram consideradas apenas as observações de fêmeas que pariram pelo menos uma vez e cuja saída do rebanho não foi por motivos de acidentes e/ou doença. Para a característica TPR, foram utilizadas informações de 1.630 vacas nascidas de 1960 a 1993, uma vez que todos os animais nascidos até esse ano já deixaram o rebanho. Para IDPP, IDSP e IDTP, foram utilizadas 1.246, 1.208 e 1.031 observações de fêmeas, respectivamente, nascidas de 1960 a 2002. As médias e os desvios-padrão para IDPP, IDSP, IDTP e TPR foram 1.144 \pm $105 ; 1.779 \pm 203 ; 2.345 \pm 333$ e $2.618 \pm 1.279$, respectivamente.

Foram estimadas as herdabilidades para as características IDPP, IDSP e IDTP e as correlações genéticas entre estas características e TPR. Foram também estimadas as correlações genéticas entre as características IDPP, IDSP, IDTP e TPR e as características mensuradas na entrada da primeira estação de monta: peso (PEM), peso ajustado para condição corporal (PEMA), condição corporal (CEM) e índice de tamanho corporal (ITCEM); ao primeiro parto: peso (PPP), peso ajustado para condição corporal (PPPA), condição corporal (CPP) e índice de tamanho corporal 
(ITCPP); e à idade adulta: peso (PAD), peso ajustado para condição corporal (PADA), condição corporal (CAD) e índice de tamanho corporal (ITCAD).

Para obtenção dos índices de tamanho corporal, o peso da fêmea foi ajustado pelo método de quadrados mínimos para idade da fêmea em dias (regressão linear), dentro de cada grupo de condição corporal. Para obtenção dos índices, o peso ajustado foi padronizado calculando-se o valor de $\boldsymbol{z}$ dentro de cada grupo de condição corporal (4, 5 e 6$)$ e nos seguintes momentos: à entrada da primeira monta, ao primeiro parto e à idade adulta. Em razão do baixo número de observações, animais com condição menor que 4 ou maior que 6 não foram considerados nas análises. Para obtenção dos valores de $\boldsymbol{z}$, foi utilizada a seguinte fórmula:

$$
\mathrm{z}=\frac{\mathrm{x}-\overline{\mathrm{x}}}{\mathrm{sd}}
$$

em que: $\mathrm{x}=$ peso da vaca $(\mathrm{kg}) ; \overline{\mathrm{x}}=$ média dos pesos das vacas dentro de grupo de condição corporal $(\mathrm{kg})$; $\mathrm{sd}=$ desvio-padrão do peso dentro de cada grupo de condição corporal.

Os valores de $\boldsymbol{z}$ maiores que $+2,5$ e menores que $-2,5$ foram eliminados por serem considerados outliers. Posteriormente, para cada estádio de vida da vaca, os valores dos três grupos de condição corporal (4, 5 e 6 ) foram agrupados em um só conjunto de dados, uma vez que os pesos estavam padronizados. Para facilitar a interpretação dos dados, foram somadas 3,5 unidades a cada valor de $\boldsymbol{z}$. Baldi (2006) estimou os parâmetros genéticos para os índices de tamanho corporal utilizando o mesmo conjunto de dados.

Foram utilizadas 1.221, 898, 914, 895, 1.095, 736, 715, 702, 1.158, 780, 789 e 776 observações de PEM, PEMA, CEM, ITCEM, PPP, PPPA, CPP, ITCPP, PAD, PADA, CAD e ITCAD, respectivamente, de animais nascidos de 1964 a 2002. As médias e os desvios-padrão de PEM, PEMA, CEM, ITCEM, PPP, PPPA, CPP, ITCPP, PAD, PADA, CAD e ITCAD foram $345 \pm 40 ; 347 \pm 33 ; 5,2 \pm 0,6 ; 3,46 \pm 0,9$; $426 \pm 51 ; 437 \pm 45 ; 4,9 \pm 0,7 ; 3,46 \pm 0,9 ; 500 \pm 65 ; 510 \pm 51$; $5,3 \pm 0,7$ e 3,46 $\pm 0,9$, respectivamente.

Para obtenção do PEMA, PPPA e PADA, os pesos foram ajustados para condição corporal da fêmea pelo método de quadrados mínimos utilizando-se a condição corporal como co-variável no modelo. O peso adulto (PAD) das fêmeas foi considerado como o peso logo após o parto para vacas de 4, 5, 6 a 8 e $>9$ anos de idade. Foi considerado apenas um peso para cada vaca adulta e, no caso de vacas com mais de um peso, utilizou-se o peso mais próximo de 6 anos, idade em que o animal provavelmente já parou de crescer. Para PADA, foi considerado o mesmo critério que para PAD, porém, foram considerados somente os animais com registro de peso e condição corporal.

Os parâmetros genéticos foram obtidos por meio da estimação dos componentes de variância e de co-variância pelo método de máxima verossimilhança restrita livre de derivadas utilizando-se o programa computacional MTDFREML (Boldman et al., 1993). O modelo estatístico adotado, representado na forma matricial, foi:

$$
\mathrm{y}=\mathrm{Xb}+\mathrm{Zg}+\mathrm{e}
$$

em que: $\mathrm{y}$ = vetor das variáveis dependentes; $\mathrm{b}$ = vetor de efeitos fixos de grupo de contemporâneos; $g$ = vetor de efeitos genéticos aditivos diretos; e = vetor de erros aleatórios residuais associados às observações; e $\mathrm{X}$ e $\mathrm{Z}=$ matrizes de incidência para os efeitos fixos e aleatórios aditivos diretos, respectivamente.

As pressuposições da distribuição de y, g e e podem ser descritas como:

$$
\left[\begin{array}{l}
y \\
g \\
e
\end{array}\right] \sim \mathrm{N}\left\{\left[\begin{array}{r}
\boldsymbol{X} \beta \\
\boldsymbol{0} \\
\boldsymbol{0}
\end{array}\right],\left[\begin{array}{ccc}
\boldsymbol{Z G Z ^ { \prime }}+\boldsymbol{R} & \boldsymbol{Z G} & \boldsymbol{R} \\
\mathbf{G} \mathbf{Z}^{\prime} & \boldsymbol{G} & \Phi \\
\boldsymbol{R} & \Phi & \boldsymbol{R}
\end{array}\right]\right\}
$$

em que $\boldsymbol{G}$ é a matriz de variâncias e co-variâncias dos efeitos aleatórios do vetor g e $\boldsymbol{R}$, a matriz de variâncias e co-variâncias residuais.

Esse modelo foi aplicado para todos os caracteres estudados; houve variação apenas dos efeitos fixos, que variaram de acordo com a característica em análise. Para a estimação de componentes de variância e estimação da herdabilidade de cada variável, foram realizadas análises unicaracterísticas e, para a estimação das correlações genéticas entre as características, foram feitas análises bicaracterísticas. As médias das variâncias genéticas e fenotípicas para cada característica, obtidas nas análises bicaracterísticas, foram usadas para estimação da herdabilidade, que, posteriormente, foi comparada à estimativa de herdabilidade obtida pela análise unicaracterística. Foi considerado em todas as análises o critério de convergência de $10^{-9}$.

O grupo de contemporâneos (GC) foi definido da seguinte forma: para IDPP, por ano-mês de nascimento da fêmea; para IDSP, por ano do primeiro parto; para IDTP, por ano-mês do segundo parto; e para TPR, por ano-mês de nascimento da fêmea. Foram considerados GC com no mínimo duas observações. Os grupos de contemporâneos para as características PEM, CEM, PEMA, ITCEM, PPP, PPPA, CPP, ITCPP, PAD, PADA, CAD e ITCAD foi definido 
por ano e mês de início da primeira estação de monta, para as quatro primeiras, e por ano e mês de parto, para as oito últimas. Em todas as análises, utilizou-se um arquivo de pedigree contendo identificação do animal, do pai e da mãe, totalizando 12.311 animais na matriz de parentesco.

\section{Resultados e Discussão}

Para todas as características estudadas, as estimativas de herdabilidade obtidas pelas análises bicaracterísticas foram semelhantes às estimativas de herdabilidade obtidas pelas análises unicaracterísticas (Tabela 1).

A estimativa de herdabilidade obtida para IDPP $(0,10)$ foi semelhante aos valores obtidos por Silva et al. (2000), Mello et al. (2006) e Castro-Pereira et al. (2007) e inferior aos relatados por Talhari et al. (2003), para a raça Canchim, de 0,12; 0,12; 0,09 e 0,23, respectivamente. Na raça Nelore, existem comunicações de herdabilidade para idade ao primeiro parto que variam de 0,04 a 0,12 (Gressler et al., 2000; Pereira et al., 2000, 2001; Dias et al., 2004). Pereira et al. (2002) obtiveram na raça Nelore estimativas de herdabilidade da idade ao primeiro parto de 0,19 e 0,02 quando os animais foram expostos pela primeira vez aos 14 e aos 26 meses de idade, respectivamente. Koots et al. (1994), em trabalho de revisão com a maioria das raças européias, relataram média de herdabilidade de IDPP de 0,14.

O valor de herdabilidade estimado $(0,08)$ para IDSP neste trabalho foi inferior ao relatado por Talhari (2002), 0,19, e semelhante ao obtido por Silva et al. (2000), 0,04. Para IDTP, o coeficiente de herdabilidade estimado $(0,14)$ também foi baixo, o que é normalmente observado nas características reprodutivas.

As herdabilidades obtidas neste trabalho para IDPP, IDSP e IDTP foram baixas e sugerem pouco progresso genético pela seleção. Segundo Dias et al. (2004), ao utilizar IDPP, apenas as fêmeas que pariram fazem parte das análises, assim, parte da variação na característica não tem como ser estimada. Também a existência de estação de monta definida pode reduzir a expressão de diferenças genéticas entre os animais. Além disso, neste trabalho, os critérios (peso e idade) usados para colocar as novilhas em reprodução podem, em parte, ter sido responsáveis pela baixa estimativa de herdabilidade obtida, pois não houve desafio das novilhas.

A herdabilidade obtida para TPR $(0,06)$ foi semelhante às relatadas por Gianlorenço et al. (2002) e Mello et al. (2002), de 0,11 e 0,12, respectivamente, obtidas para a raça Canchim pelo método REML e análises bicaracterísticas com o peso e o perímetro escrotal de machos ao ano de idade e peso de fêmeas ao desmame e ao ano de idade, respectivamente. No entanto, esses mesmos autores, utilizando análise unicaracterística e inferência Bayesiana, obtiveram herdabilidades superiores ( 0,33 e 0,22 , respectivamente). A herdabilidade obtida neste trabalho para TPR sugere que esta característica dificilmente pode ser melhorada por meio de seleção. Além disso, a expressão tardia desta característica aumentaria o intervalo de gerações e diminuiria o ganho genético anual.

As correlações genéticas de TPR com IDPP, IDSP e IDTP foram negativas e baixas (Tabela 2). No entanto, as correlações entre as idades foram altas e positivas. As magnitudes das correlações entre TPR e as idades indicam que estas características são pouco relacionadas. Mercadante et al. (2000) e Mello et al. (2006) obtiveram correlações entre IDPP e TPR que corroboram as obtidas neste estudo. Bertazzo et al. (2004), no entanto, estimaram correlação positiva e baixa entre IDPP e TPR.

Em termos gerais, as magnitudes das correlações entre os pesos e as idades ao parto variaram de baixas a nulas (Tabela 3), enquanto a correlação de PPP e IDSP foi positiva. Segundo Rovira (1996), vacas de maior peso apresentam maiores requerimentos nutricionais para manutenção, o que influencia negativamente no reinício da atividade reprodutiva após o parto quando as exigências nutricionais não são atendidas, sobretudo nas vacas primíparas. No entanto, de acordo com os resultados obtidos, a seleção de fêmeas para maior PPP terá pouca influência sobre a idade ao segundo parto, o que é favorável, uma vez que o maior

Tabela 1 - Estimativas dos componentes de variância genética aditiva direta $\left(\hat{\sigma}_{a}^{2}\right)$, residual $\left(\hat{\sigma}_{e}^{2}\right)$ e fenotípica $\left(\hat{\sigma}_{p}^{2}\right)$ e dos coeficientes de herdabilidade $\left(\hat{h}^{2}\right)$, obtidas em análises unicaracterísticas, e valores médio $\left(\hat{h}_{b}{ }^{2}\right)$, máximo $\left(\max \hat{h}_{b}{ }^{2}\right)$ e mínimo $\left(\min \hat{h}_{b}{ }^{2}\right)$ dos coeficientes de herdabilidade obtidos em análises bicaracterísticas

\begin{tabular}{lccccccc}
\hline Característica $^{1}$ & $\hat{\sigma}_{a}^{2}$ & $\hat{\sigma}_{e}^{2}$ & $\hat{\sigma}_{p}^{2}$ & $\hat{h}^{2}$ & $\hat{h}_{b}^{2}$ & $\max _{b}^{2}$ & $\hat{\min }_{b}^{2}$ \\
\hline IDPP & 1.105 & 9.941 & 11.046 & $0,10 \pm 0,05$ & 0,11 & 0,13 \\
IDSP & 2.437 & 27.586 & 30.023 & $0,08 \pm 0,05$ & 0,08 & 0,10 \\
IDTP & 8.038 & 48.915 & 56.952 & $0,14 \pm 0,06$ & 0,15 & 0,17 \\
TPR & 93.358 & 1.396 .337 & 1.489 .695 & $0,06 \pm 0,00$ & 0,07 & 0,08 & 0,14 \\
\hline
\end{tabular}

1 Tempo de permanência no rebanho (TPR), idades ao primeiro (IDPP), segundo (IDSP) e terceiro (IDTP) partos. 
Tabela 2 - Estimativas de co-variâncias $\left(\sigma_{\mathrm{a} 1 \mathrm{a} 2}\right)$ e das correlações genéticas ( $\left.\mathrm{r}_{\mathrm{a} 1 \mathrm{a} 2}\right)$ das idades ao primeiro (IDPP), segundo (IDSP) e terceiro (IDTP) partos com o tempo de permanência no rebanho (TPR), em análises bicaracterísticas

\begin{tabular}{|c|c|c|c|c|c|c|}
\hline \multirow[b]{3}{*}{ Característica } & \multicolumn{6}{|c|}{ Característica } \\
\hline & \multicolumn{2}{|c|}{ IDPP } & \multicolumn{2}{|c|}{ IDSP } & \multicolumn{2}{|c|}{ IDTP } \\
\hline & $\sigma_{\mathrm{a} 1 \mathrm{a} 2}$ & $\mathrm{r}_{\mathrm{a} 1 \mathrm{a} 2}$ & $\sigma_{\mathrm{a} 1 \mathrm{a} 2}$ & $\mathrm{r}_{\mathrm{a} 1 \mathrm{a} 2}$ & $\sigma_{\mathrm{a} 1 \mathrm{a} 2}$ & $\mathrm{r}_{\mathrm{a} 1 \mathrm{a} 2}$ \\
\hline IDPP & - & - & 1.047 & 0,83 & 1.846 & 0,88 \\
\hline IDSP & - & - & - & - & 3.734 & 1,00 \\
\hline TPR & -1.294 & $-0,20$ & -3.953 & $-0,28$ & -529 & $-0,02$ \\
\hline
\end{tabular}

Tabela 3 - Estimativas das correlações genéticas das características estudadas em análises bicaracterísticas

\begin{tabular}{lrrrr}
\hline & \multicolumn{4}{c}{ Característica } \\
\cline { 2 - 5 } Característica & \multicolumn{1}{c}{ IDPP } & IDSP & IDTP & TPR \\
\hline PEM & $-0,17$ & $-0,07$ & $-0,03$ & $-0,30$ \\
P P P & 0,06 & 0,35 & 0,12 & $-0,29$ \\
PAD & $-0,01$ & 0,32 & 0,28 & $-0,46$ \\
CEM & $-0,12$ & $-0,16$ & 0,08 & 0,27 \\
CPP & $-0,73$ & $-0,10$ & $-0,12$ & 0,61 \\
CAD & 0,08 & 0,37 & 0,23 & $-0,04$ \\
ITCEM & $-0,10$ & $-0,35$ & $-0,38$ & $-1,00$ \\
ITCPP & 0,44 & 0,20 & 0,17 & $-0,88$ \\
ITCAD & 0,19 & 0,44 & 0,45 & $-1,00$ \\
PEMA & $-0,15$ & $-0,22$ & $-0,19$ & $-1,00$ \\
P P P A & 0,42 & 0,51 & 0,28 & $-0,83$ \\
PADA & 0,23 & 0,35 & 0,38 & $-1,00$ \\
\hline
\end{tabular}

IDPP = idade ao primeiro parto; IDSP = idade ao segundo parto; IDTP = idade ao terceiro parto; TPR = tempo de permanência no rebanho; $P E M=$ peso à primeira monta; $\mathrm{PPP}=$ peso ao primeiro parto; $\mathrm{PAD}=$ peso à idade adulta; $\mathrm{CEM}=$ condição corporal à primeira monta; $\mathrm{CPP}=$ condição corporal ao primeiro parto; $C A D=$ condição corporal à idade adulta; ITCEM = índice de tamanho corporal à primeira monta; ITCPP = índice de tamanho corporal ao primeiro parto; ITCAD = índice de tamanho corporal à idade adulta; PEMA = peso ajustado para condição corporal à primeira monta; peso ajustado para condição corporal ao primeiro parto; PADA = peso ajustado para condição corporal à idade adulta.

peso da vaca primípara parece não comprometer a reconcepção após o primeiro parto.

A correlação entre PAD e IDPP obtida neste trabalho foi praticamente nula. Winkler et al. (1993) e Mello et al. (2006) relataram estimativas de 0,16 e 0,19 para as mesmas características. A correlação genética positiva entre PAD e IDSP neste estudo foi maior que a obtida por Winkler et al. (1993), 0,10.

Entre PEM e IDPP, a correlação obtida neste trabalho $(-0,17)$ corrobora as relatadas na literatura (Mercadante et al., 2000; Silva et al., 2000; Talhari et al., 2003; Castro-Pereira et al., 2007), em que, a idades inferiores a um ano, o peso e a IDPP estão associados negativa e favoravelmente, apesar dos baixos valores. É possível que os animais mais desenvolvidos entrem em reprodução mais cedo, determinando menor idade ao primeiro parto. Além disso, o critério de peso vivo normalmente utilizado para entrada das novilhas em reprodução pode favorecer as novilhas mais desenvol- vidas que terão mais chances de conceber em idade mais jovem. Ao segundo e ao terceiro parto, as correlações obtidas com PEM são praticamente nulas. Talhari (2002) verificou correlações genéticas favoráveis de medidas de comprimento, altura e perímetro torácico, do porte e da área corporal com a idade ao primeiro parto. Entretanto, com a idade ao segundo parto, houve redução na magnitude das estimativas ou mesmo troca de sinal, passando a ser próximas de zero ou desfavoráveis.

Observa-se, portanto, que à idade jovem, ou seja, à entrada da primeira estação de monta, a correlação genética do peso com a idade ao primeiro parto, apesar de baixa, é favorável, tornando-se nula com as idades aos partos seguintes. Esse resultado parece lógico, pois o animal mais desenvolvido em idade jovem concebe mais jovem e pare mais jovem. Entretanto, após o primeiro parto, o animal mais pesado, portanto mais exigente, apresentará maior período de serviço. Assim, a vantagem apresentada ao primeiro parto começa a desaparecer já ao segundo parto, fato reforçado pelas correlações genéticas entre PPP e PAD e as idades aos partos. Nesses casos, as correlações genéticas entre os pesos e a idade ao primeiro parto são nulas e passam a positivas (portanto, desfavoráveis), apesar de baixas, ao segundo parto, sugerindo que animais mais pesados apresentam maior período de serviço após o parto.

As correlações genéticas das características PEM e PPP com TPR foram baixas, enquanto a de PAD e TPR foi moderada, todas desfavoráveis, sugerindo que as fêmeas mais pesadas são menos longevas ou permanecem por menos tempo no rebanho. O peso corporal está diretamente associado às necessidades energéticas para manutenção (NRC, 1996), o que, em condições de baixa oferta de forragem, traz desequilíbrio nutricional maior para as vacas de maior peso e, em conseqüência, atraso no reinício da atividade reprodutiva após o parto (Short \& Adams, 1988). Portanto, as vacas de maior peso possivelmente apresentam anestro pós-parto maior, retardando a reconcepção após o parto e diminuindo a possibilidade de conceber antes do fim da estação de monta. Mello et al. (2006), utilizando inferência bayesiana, relataram correlação negativa, mas 
de baixa magnitude, entre PAD e TPR. Bertazzo et al. (2004), utilizando pesos aos 550 dias de idade em fêmeas da raça Nelore, obtiveram correlação negativa e moderada com TPR. No entanto, Mercadante et al. (2000), Gianlorenço et al. (2002), Mello et al. (2002) e Gianlorenço et al. (2003), utilizando pesos a idades jovens, obtiveram valores de correlação positivos e de baixa a moderada magnitude. Parece que a idades jovens, o peso corporal e a longevidade apresentam associação positiva, o que pode ser revertido em idades avançadas, como verificado neste trabalho e nos trabalhos utilizando pesos a idades adultas.

Com exceção da correlação de CPP com IDPP, as demais correlações entre condição corporal e idades ao parto foram baixas (Tabela 3). A correlação entre CPP e IDPP indica associação favorável entre as características. As correlações entre CEM e CAD com TPR foram baixa e nula, respectivamente. A CPP apresentou correlação moderada e positiva com TPR, indicando que a seleção para maior CPP aumentará o tempo de permanência da fêmea no rebanho. Mello et al. (2006) obtiveram correlação quase nula entre CPP e TPR utilizando inferência Bayesiana. Segundo Pigurina (2000), as vacas de primeira cria que não reconcebem após o primeiro parto constituem alta porcentagem dos refugos do rebanho, em razão de suas altas exigências nutricionais por se encontrarem em fase de crescimento. Segundo Bellows \& Short (1978), as vacas com baixa condição corporal ao parto apresentam anestro pós-parto mais prolongado, demorando a reconcepção e, portanto, atrasando o parto seguinte. Provavelmente, as vacas de primeira cria com maior condição corporal tiveram anestro pós-parto menor e maior possibilidade de conceber antes do fim da estação de monta. Portanto, não foram descartadas por falha reprodutiva e permaneceram por maior tempo no rebanho.

As correlações entre os índices de tamanho corporal com IDPP, IDSP e IDTP (Tabela 3) apresentaram tendências semelhantes às obtidas entre estas características e os pesos, mas as magnitudes das correlações obtidas com os índices foram maiores. As correlações entre os índices de tamanho corporal e o tempo de permanência foram muito altas, provavelmente em razão de problemas na estrutura dos dados. De acordo com Baldi (2006), que analisou o mesmo conjunto de dados, esses problemas ocorrem porque mais da metade das observações correspondem a vacas com escore de condição corporal igual a 5, o que afeta a interpretação dos índices de tamanho corporal e sua associação com as outras características.

Entre os pesos ajustados e as características reprodutivas e de longevidade, as correlações foram semelhantes às obtidas entre os índices de tamanho corporal e essas características. Isso sugere que o peso ajustado para condição corporal e os índices de tamanho corporal apresentam associação genética semelhante com as características reprodutivas e de longevidade das fêmeas.

\section{Conclusões}

No rebanho estudado, espera-se que os ganhos genéticos obtidos pela seleção para o tempo de permanência no rebanho e para as idades ao primeiro, segundo e terceiro partos sejam baixos. O aumento no peso à idade adulta deverá acarretar diminuição da vida produtiva da fêmea no rebanho. A seleção para maior peso da fêmea à primeira monta, ao primeiro parto e à idade adulta não deve alterar significativamente as idades ao primeiro, segundo e terceiro partos. Espera-se que mudanças nos índices de tamanho corporal ou nos pesos ajustados para condição corporal, por meio da seleção, apresentem repostas correlacionadas para as características reprodutivas e de longevidade com tendências semelhantes, mas de maior magnitude, em relação à seleção para peso não-ajustado.

\section{Literatura Citada}

ALENCAR, M.M. Critérios de seleção em bovinos de corte no Brasil. In: SIMPOSIO NACIONAL DE MELHORAMENTO ANIMAL, 4., 2002, Campo Grande. Anais... Campo Grande: Sociedade Brasileira de Melhoramento Animal, 2002. (CD-ROM).

BALDI, F.S. Relação genética de características de tamanho corporal com características de eficiência reprodutiva e produtiva de fêmeas da raça Canchim. Jaboticabal: Universidade Estadual Paulista, 2006. 97p. Dissertação (Mestrado em Genética e Melhoramento Animal) - Universidade Estadual Paulista, 2006.

BELLOWS, R.A.; SHORT, R.E. Effects of pre-calving feed level on birth weight, calving difficulty and subsequent fertility. Journal of Animal Science, v.46, p.1522-1530, 1978.

BERTAZZO, R.P.; FREITAS, R.T.F.; GONCALVES, T.M. Parâmetros genéticos de longevidade e produtividade de fêmeas da raça Nelore. Revista Brasileira de Zootecnia, v.33, n.5, p.1118-1127, 2004.

BOLDMAN, K.G.; KRIESE, L.A.; van VLECK, L.D. et al. A manual for use of MTDFREML. Clay Center: USDA-ARS, 1993. 120p.

CASTRO-PEREIRA, V.M.; ALENCAR, M.M.; BARBOSA, P.F. Estimativas de parâmetros genéticos e de ganhos direto e indireto à seleção para características de crescimento de machos e fêmeas da raça Canchim. Revista Brasileira de Zootecnia, v.36, n.4, p.1037-1044, 2007 (supl.).

DIAS, L.T.; EL FARO, L.; ALBUQUERQUE, L.G. Heritability estimates for age at first calving in Nelore cattle. Revista Brasileira de Zootecnia, v.33, n.1, p.97-102, 2004.

FERREL, C.L.; JENKINS, T.G. Cow type and the nutritional environment: nutritional aspects. Journal of Animal Science, v.61, n.3, p.725-741, 1985.

GIANLORENÇO, V.K.; ALENCAR, M.M.; MELLO, S.P. Correlações genéticas entre peso e perímetro escrotal de machos com o tempo de permanência de fêmeas em um rebanho da raça Canchim In: SIMPÓSIO NACIONAL DE MELHORAMENTO ANIMAL, 4., 2002, Campo Grande. Anais... Campo Grande: Sociedade Brasileira de Melhoramento Animal, 2002. p-96. 
GIANLORENCO, V.K.; ALENCAR, M.M.; TORAL, F.L.B. Herdabilidades e correlações genéticas de características de machos e fêmeas, em um rebanho bovino da raça Canchim. Revista Brasileira de Zootecnia, v.32, n.6, p.1587-1593, 2003 (suppl.1).

GRESSLER, S.L.; BERGMANN, J.A.G.; PENNA, V.M. et al. Estudo das associações genéticas entre perímetro escrotal e características reprodutivas de fêmeas Nelore. Revista Brasileira de Zootecnia, v.29, n.2, p.427-437, 2000.

JOHNSTON, D.J.; BUNTER, K.L. Days to calving in Angus cattle: Genetic and environmental effects, and covariances with other traits. Livestock Production Science, v.45, n.1, p.13-22, 1996.

KOOTS, K.R.; GIBSON, J.P.; SMITH, C. et al. Analyses of published genetic parameter estimates for beef production traits. 1 . Heritability. Animal Breeding Abstract, v.62, p.309-338, 1994.

KRESS, D.D.; HAUSER, E.R.; CHAPMAN, A.B. Efficiency of production and cow size in beef cattle. Journal of Animal Science, v.29, n.2, p.373-383, 1969.

MELLO, S.P.; ALENCAR, M.M.; TORAL, F.L.B. et al. Estimativas de parâmetros genéticos para características de crescimento e produtivas em vacas da raça Canchim, utilizando-se inferência bayesiana. Revista Brasileira de Zootecnia, v.35, n.1, p.9297, 2006.

MELLO, S.P.; GIANLORENÇO, V.K.; ALENCAR, M.M. et al. Correlações genéticas entre pesos e tempo de permanência de fêmeas em um rebanho da raça canchim In: SIMPÓSIO NACIONAL DE MELHORAMENTO ANIMAL, 4., 2002, Campo Grande. Anais... Campo Grande: Sociedade Brasileira de Melhoramento Animal, 2002. p.96-98.

MERCADANTE, M.E.Z.; LÔBO, R.B.; OLIVEIRA, H.N. Estimativas de (co)variâncias entre características de reprodução e de crescimento em fêmeas de um rebanho Nelore. Revista Brasileira de Zootecnia, v.29, n.4, p.997-1004, 2000.

MERCADANTE; M.E.Z.; PACKER, I.U.; RAZOOK, A.G. et al. Direct and correlated responses to selection for yearling weight on reproductive performance of Nelore cows. Journal of Animal Science, v.81, p.376-384, 2003.

MORRIS, C.A.; WILTON, J.W. The influence of body size on the economic efficiency of cows. A review. Animal Breeding Abstract, v.45, n.3, p.139, 1977.

NORTHCUTT, S.L.; WILSON, D.E. Genetic parameter estimates and expected progeny differences for mature size in Angus cattle. Journal Animal Science, v.71, p.1148-1153, 1993.

NATIONAL RESEARCH COUNCIL - NRC. Nutrient requirements of beef cattle. 7.ed. Washington, D.C.: National Academy Press, 1996. 242p.
PEREIRA, E.; ELER, J. P.; COSTA, F.A.A. et al. Análise genética da idade ao primeiro parto e do perímetro escrotal em bovinos da raça Nelore. Arquivo Brasileiro de Medicina Veterinária e Zootecnia, v.53, n.1, p.116-121, 2001.

PEREIRA, E.; ELER, J.P.; FERRAZ, J.B.S. Correlação genética entre perímetro escrotal e algumas características reprodutivas na raça Nelore. Revista Brasileira de Zootecnia, v.29, n.6, p.1676-1683, 2000.

PEREIRA, E.; ELER, J.P.; FERRAZ, J.B.S. Análise genética de características reprodutivas na raça Nelore. Pesquisa Agropecuária Brasileira, v.37, n.5, p.703-708, 2002.

PIGURINA, G. Situación de la cría en el Uruguay. In: Instituto Nacional de Investigación Agropecuaria (Ed.) Estrategia para acortar el anestro posparto en vacas de carne. Montevideo: INIA, 2000. p.1-6. (Serie Técnica, 108).

ROVIRA, J. Manejo nutritivo de los rodeos de cría en pastoreo. Montevideo: Hemisferio Sur, 1996. 288p.

SHORT, R.E.; ADAMS, D.C. Nutritional and hormonal interrelationships in beef cattle reproduction. Canadian Journal of Animal Science, v.68, p.29-42, 1988.

SILVA, A.M.; ALENCAR, M.M.; FREITAS, A.R. Herdabilidade e correlações genéticas para peso e perímetro escrotal de machos e características reprodutivas e de crescimento de fêmeas, na raça Canchim. Revista Brasileira de Zootecnia, v.29, p.22232230, 2000.

TALHARI, F.M. Parâmetros genéticos e fatores de meio para medidas corporais e características reprodutivas de fêmeas das raças Canchim e Santa Gertrudis. São Carlos: Universidade Federal de São Carlos , 2002. 54p. Dissertação de Mestrado (Genética e Evolução) - Universidade Federal de São Carlos, 2002.

TALHARI, F.M.; ALENCAR, M.M.; MASCIOLI, A.S. Correlações genéticas entre características produtivas das fêmeas em um rebanho da raça Canchim. Revista Brasileira de Zootecnia, v.32, n.4, p.880-886, 2003.

VARGAS, C.A.; OLSON, T.A.; CHASE, C.C. et al. Influence of frame size and body score on performance of Brahman cattle. Journal of Animal Science, v.77, p.140-3149, 1999.

WILTBANK, J.N.; ROWDEN, W.W.; INGALLS, J.E. et al. Effect of energy level on reproductive phenomena of mature Hereford cows. Journal of Animal Science, v.21, p.219-226, 1962.

WINKLER, R.; PENNA, V.M.; PEREIRA, C.S. et al. Estudo das relações entre o tamanho corporal e algumas características reprodutivas em fêmeas bovinas adultas da raça Guzerá. In: REUNIÃO ANUAL DA SOCIEDADE BRASILEIRA DE ZOOTECNIA, 30., 1993, Rio de Janeiro. Anais... Rio de Janeiro: Sociedade Brasileira de Zootecnia, 1993. p.292. 